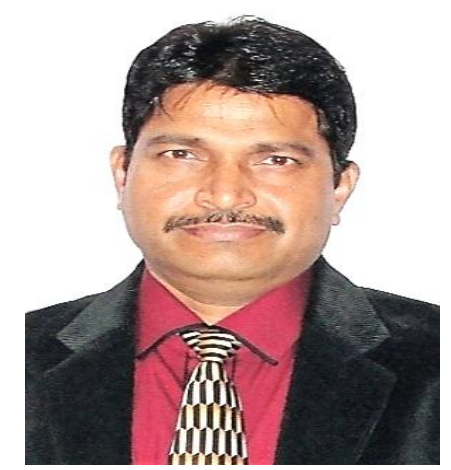

Dear Friends

Season's Greetings !!

Diabetes is a chronic debilitating disorder that has emerged as an epidemic in both worlds. Both the prevalence and incidence of diabetes continues to rise sparing no country. Diabetes is the most important threat to public health in this century owing to its detrimental effect on the micro and macro vasculature with effects on every organ in the body. ${ }^{1}$ The incidence of type 2 diabetes has been on rise dramatically due to increased life expectancy along with sedentary lifestyles and obesity. In 2009, the International Diabetes Federation has launched a 5-year programme on education and prevention. Yearly there are 4 million deaths worldwide due to diabetes. They estimated that 285 million people across the world are living with diabetes; an estimated $70 \%$ are in low-income and middle-income countries (LMIC). Around $90 \%$ of the burden is caused by type 2 diabetes, which is a preventable chronic disease.

Diabetic macular edema (DME) is important cause of visual disability in diabetics. The Early Treatment Diabetic Retinopathy Study (ETDRS) defined macular edema as the thickening of the retina and/or hard exudates within 500 micron of the center of the macula. ${ }^{2}$ In the Wisconsin Epidemiologic Study of Diabetic Retinopathy (WESDR), the 10-year rate of developing DME was $20.1 \%$ in patients with type 1 diabetes and, in type 2 diabetes, it was $25.4 \%$ for those treated with insulin, and $13.9 \%$ for those not treated with insulin.

The pathogenesis of DME involves systemic as well as local risk factors. These risk factors may alter the blood-retinal barrier allowing the leakage of proteins and fluid into the macula. Systemic risk factors associated with DME include male gender, age, systolic blood pressure, proteinuria,hyperlipidemia, insulin and diuretic use, a longer duration of diabetes, higher $\mathrm{HbA1c}$ levels and pan-retinal photocoagulation (PRP). ${ }^{3-}$

Local factors that may influence DME include angiogenic factors, such as vascular endothelial growth factor (VEGF), protein kinase C (PKC), prostaglandins, growth hormone, and the anatomy of the posterior hyaloid face.

Clinically-significant macular edema (CSME) is defined as one or more of the following: retinal thickening at or within 500 micron of the center of the macula; hard exudates at or within 500 micron of the center of the macula, if associated with adjacent retinal thickening; or a zone or zones of retinal thickening one disc area in size, at least part of which is within one disc diameter of the center of the macula. This definition primarily refers to eyes eligible for laser photocoagulation. With the advent of medical management (anti-VEGF agents or corticosteroids) DME is more appropriately defined as center-involving versus non-center involving, with most cases of centerinvolved macular edema appropriately eligible for treatment with pharmacotherapy. Intravenous fluorescein angiography (FA) and optical coherence tomography $(\mathrm{OCT})^{7}$ can assist in the evaluation of DME.

The treatment of DME is focused on optimizing systemic risk factors and use of laser, pharmacologic, or surgical modalities to reduce leakage into the macula and subsequent macular edema.

\section{Systemic Therapies}

The United Kingdom Prospective Diabetes Study (UKPDS) of type 2 diabetics, ${ }^{8}$ the Diabetes Control and Complications Trial (DCCT) of type 1 diabetics, ${ }^{9,10}$ and the WESDR ${ }^{11}$ established that a higher level of glycosylated hemoglobin is a risk factor for DME. The DCCT and UKPDS established that intensive control of blood glucose should be a goal for almost all patients with diabetes. One study showed that treatment of hyperlipidemia in patients with macular edema and hard exudates resulted in improvement or stability of visual acuity. Treatment of renal dysfunction, anaemia or smoking cessation has not been proven to have a direct benefit on DME; however, they are still encouraged for diabetic patients. 
The effect of VEGF on retinal vascular permeability appears to be mediated predominantly by the beta-isoform of Protein Kinase C. ${ }^{12}$ Ruboxistaurin (RBX) is an orally administered, isoform-selective inhibitor of PKC-beta. Inflammation plays a role in DME and chronic inflammation has been shown to be mediated, in part, by the cyclooxygenase (COX) isozymes localized in the retina. Immunohistochemistry studies and animal models of retinopathy have shown the efficacy of COX-2 inhibitors. Potential side effects of COX-2 inhibitors include allergic reactions, gastrointestinal discomfort, and gastrointestinal bleeding.

\section{Ocular Therapies}

The mechanism of action of laser photocoagulation is unknown. The possible explanations include laserinduced destruction of the oxygen-consuming peripheral retina and increased diffusion of oxygen through the laser scars to the inner retina. ${ }^{13}$ The ETDRS has described two methods of treatment with laser photocoagulation: focal or grid-pattern. Focal photocoagulation consisted of whitening or darkening of microaneurysms and areas of focal fluorescein leakage with 50-200 micron laser spots. Grid-pattern photocoagulation consisted of light burns with a spot size of 50-200 micron, spaced at least one spot size apart in an area more than 500micron from the foveal center and the optic disc. Retreatment was carried out if CSME was present at the 4-month follow-up visit. FA was performed to detect new or residual areas of focal or diffuse leakage. Focal leaks within 500 micron from the center were treated if the visual acuity was worse than 20/40, and if such treatment could be performed without significant risk to the center of the fovea .Laser photocoagulation of the macula can cause chorioretinal scars to expand up to $300 \%$ and produce dense focal scotomas. Other potential adverse effects include reduced color vision, choroidal neovascularization, retinal pigment epithelium (RPE) fibrous metaplasia, and inadvertent photocoagulation of the center of the macula.

Alternative laser delivery systems are being developed to reduce complications of focal photocoagulation. The micro-pulsed laser system is able to achieve the desired benefits by delivering energy as a train of short bursts to the RPE with sufficient time between bursts to allow the heated tissue temperature to return to normal. This technique reduces collateral damage significantly and decreases the risk of expansion of retinal scars. Solid-state green patternscanning laser (PASCAL) with a short-duration $(0.01 \mathrm{~s})$ has also shown comparable results with standard focal laser treatment for DME after a 4-month follow-up. Furthermore, to improve the treatment accuracy and localization of retinal lesions, a navigated laser (NAVILAS_; OD-OS GmbH, Teltow, Germany) photocoagulator has been developed. It consists of a retinal eye-tracking laser delivery system with integrated digital fundus imaging that allows overlay of a treatment plan, based on either fundus photography or FA, onto a real-time image of the patient's retina. The other advantages are: a larger area of the retina can be visualized than with a slit lamp, images are reflex free, infrared fundus illumination, no requirement for contact lens use or topical anesthesia during the procedure, and the availability of an immediate detailed report.

Although focal laser photocoagulation per ETDRS was successful in reducing the rates of visual loss due to DME, many patients did not recover lost vision and there was a subset of patients who were unresponsive to this therapy. This led to the use of intraocular pharmacologic agents for management of DME. Corticosteroids decrease the release of prostaglandins and inhibit the expression of the VEGF gene. ${ }^{14,15}$ These anti-inflammatory and anti-VEGF properties may be able to reduce breakdown of the blood-retina barrier. The various routes used for corticosteroids delivery in the treatment of DME include periocular injection, intravitreal injection, or via the implantation of a biodegradable or nonbiodegradable sustained-release device. One potential drawback of intravitreal injection is that the treatment effect typically wanes, and patients that are initially responsive to treatment may require repeated injections. An extended-release product could reduce the risks associated with repeated injections. Retisert (Bausch \& Lomb, Rochester, NY, USA) is FDA-approved for the treatment of uveitis and is designed to release $0.59 \mathrm{~g} /$ day fluocinolone acetonide. It has been evaluated for the treatment of DME and showed some promise in reducing macular thickness in DME; however, after 2 years, $80-90 \%$ of phakic patients required cataract extraction and $15-20 \%$ of patients required incisional glaucoma surgery. An intravitreal drug delivery system (DDS) that delivers dexamethasone directly to the posterior segment for 35 days is also being developed. Cataracts and elevation of intraocular pressure are the principal adverse effects from use of ocular steroids. Intravitreal injection and implantable devices carry additional risks associated with the injection and implantation procedure, respectively.

Increased vascular permeability is a hallmark of DME. In human eyes with DR, hypoxia causes upregulation of VEGF production, and leads to retinal capillary hyperpermeability. The anti-VEGF therapies available at this time include: pegaptanib sodium, ranibizumab, bevacizumab, and aflibercept. ${ }^{16}$ There are several reports of an association between DME and the anatomy of the vitreoretinal interface. An attached posterior hyaloid predisposes to the development of DME and, hence, pars plana vitrectomy may have a role in the treatment of DME. Patients with macular ischemia, RPE atrophy, subfoveal lipid, and a baseline visual acuity of $20 / 200$ or less tended to respond less favorably to surgery. The expected complications of 
vitrectomy include cataract progression, vitreous hemorrhage, and retinal tear or detachment. ${ }^{17,18}$

There has been considerable progress in understanding the pathophysiology of DME and the development of new therapies. Based on various clinical and epidemiologic studies, it is recommended for all patients with diabetes to maintain good control of blood sugars, blood pressure, and hyperlipidemia as determined by their primary care physician. Focal or grid photocoagulation remains the first-line treatment in the majority of patients with non-center involved DME. In many patients with center-involved DME, intravitreal injection of anti-VEGF therapies is becoming commonly used. Patients unresponsive to anti-VEGF therapies may benefit from intravitreal injection of triamcinolone or possibly an extendedrelease steroid delivery system to deliver corticosteroids to the posterior segment. However, patients need to understand the risks of these treatments, especially with regards to cataract and glaucoma. In patients with vitreoretinal interface disease, vitrectomy with removal of the posterior vitreous may be a viable option. As new and improved therapies are continuously developed, treatment paradigms will also change, with DME patients being the ultimate beneficiaries of these exciting developments.

\section{References}

1. Harris MI, Flegal KM, Cowie CC, Eberhardt MS, Goldstein DE, Little RR. Prevalence of diabetes, impaired fasting glucose, and impaired glucose tolerance in U.S. adults. The Third National Health and Nutrition Examination Survey, 1988-1994. Diabetes Care 1998;21(4):475-76.

2. Early Treatment Diabetic Retinopathy Study Research Group. Photocoagulation for diabetic macular edema. Early Treatment Diabetic Retinopathy Study report number 1. Arch Ophthalmol. 1985;103:1796-806.

3. Augustin A, Loewenstein A, Kuppermann BD. Macular edema. General pathophysiology. Dev Ophthalmol. 2010;47:10-26.

4. Stratton IM, Adler AI, Neil HA, et al. Association of glycaemia with macrovascular and microvascular complications of type 2 diabetes (UKPDS 35): prospective observational study. BMJ. 2000;321: 405-12.

5. Adler AI, Stratton IM, Neil HA, et al. Association of systolic blood pressure with macrovascular and microvascular complications of type 2 diabetes (UKPDS 36): prospective observational study. $B M J$. 2000;321:412-9.

6. McDonald HR, Schatz H. Visual loss following panretinal photocoagulation for proliferative diabetic retinopathy. Ophthalmology. 1985;92:388-93.

7. Al-latayfeh MM, Sun JK, Aiello LP. Ocular coherence tomography and diabetic eye disease. Semin Ophthalmol. 2010;25:192-7.

8. UK Prospective Diabetes Study Group. Tight blood pressure control and risk of macrovascular and microvascular complications in type 2 diabetes: UKPDS 38. BMJ. 1998;317:703-13. (erratum in: BMJ. 1999;318:29).

9. The Diabetes Control and Complications Trial Research Group. The effect of intensive treatment of diabetes on the development and progression of long-term complications in insulin-dependent diabetes mellitus. $N$ Engl J Med. 1993;329:977-86.

10. Diabetes Control and Complications Trial Research Group. Progression of retinopathy with intensive versus conventional treatment in the diabetes control and complications trial. Ophthalmology. 1995;102:647-61.

11. Klein R, Klein BE, Moss SE, et al. The Wisconsin epidemiologic study of diabetic retinopathy. IV. Diabetic macular edema. Ophthalmology. 1984;91:1464-74.

12. Aiello LP, Bursell SE, Clermont A, et al. Vascular endothelial growth factor-induced retinal permeability is mediated by protein kinase $\mathrm{C}$ in vivo and suppressed by an orally effective beta-isoform-selective inhibitor. Diabetes. 1997;46:1473-80.

13. Ahmadi MA, Lim JI. Update on laser treatment of diabetic macular edema. Int Ophthalmol Clin. 2009;49:87-94.

14. Nauck M, Karakiulakis G, Perruchoud AP, et al. Corticosteroids inhibit the expression of the vascular endothelial growth factor gene in human vascular smooth muscle cells. Eur J Pharmacol. 1998;341:309-15.

15. Nauck M, Roth M, Tamm M, et al. Induction of vascular endothelial growth factor by platelet-activating factor and platelet-derived growth factor is downregulated by corticosteroids. Am J Respir Cell Mol Biol. 1997;16:398406.

16. Witkin AJ, Brown GC. Update on nonsurgical therapy for diabetic macular edema. Curr Opin Ophthalmol. 2011;22:185-9. 